\title{
The effect of motor engagement on memory: Testing a motor-induced encoding account
}

\author{
Kaleb T. Kinder ${ }^{1}$ (1) Aaron T. Buss ${ }^{1}$ \\ Accepted: 23 October 2020 / Published online: 6 November 2020 \\ (C) The Psychonomic Society, Inc. 2020
}

\begin{abstract}
The motor system is traditionally thought to reflect the output of cognition. However, the inverse relationship of how the motor system impacts cognitive processes is less known. Work on this interaction has demonstrated that recognition memory for stimuli presented in combination with the inhibition of a prepared action is weaker compared to stimuli associated with the execution of an action (Chiu \& Egner, Psychological Science, 26, 27-38, 2015a). This effect has been explained through competition for common neural resources: to the extent that response inhibition processes are recruited, fewer resources are available for memory encoding (Chiu \& Egner, Journal of Neuroscience, 35, 11936-11945, 2015b). Alternatively, it has been proposed that action execution enhances memory encoding (Yebra et al., Nature Communications, 10(1), 1-12, 2019). In this report, we examined how recognition memory for stimuli paired with both the preparation and execution of a motor response compare to stimuli absent of any motor processes. We first replicated Chiu and Egner (2015a, 2015b). Next, we added a motor-neutral condition as a baseline comparison. Across three experiments, recognition memory for stimuli associated with action execution was superior to stimuli absent of motor demands. More importantly, we found that recognition memory for stimuli associated with motor preparation, but no subsequent execution, was also superior to stimuli that did not engage the motor system (Experiments 2a and $2 b$ ). These results support a motor-induced encoding effect, in which the degree of motor processing (both action preparation and action execution) enhanced memory encoding.
\end{abstract}

Keywords Motor-induced encoding $\cdot$ Memory $\cdot$ Motor engagement $\cdot$ Response inhibition $\cdot$ Go/no-go

\section{Introduction}

Goal-directed behavior requires the organization of multiple cognitive systems, guided by a collection of cognitive control processes termed executive functions (Miyake et al., 2000). Executive functions confront real-time streams of environmental demands by monitoring perceptual information for relevant stimuli and activating context-appropriate behavior via motor systems. For instance, consider the steps underlying the task of changing lanes in a vehicle. In one moment, a motor movement is selected and prepared to turn the steering wheel. However, if another vehicle were perceived in the neighboring lane just prior to the execution of this movement, it would be essential to inhibit that now unwanted action and update the goal to remain in the original lane. During each step of

Kaleb T. Kinder

kkinder5@vols.utk.edu

1 Department of Psychology, University of Tennessee, 1404 Circle Drive, Knoxville, TN 37916, USA processing illustrated above, cognitive control processes interface with perception and modulate motor processes to give rise to desired outcomes. Despite this real-time interplay, interactions between motor processes and core cognitive constructs such as attention and memory are not well understood.

One study explored this topic by investigating how response inhibition, an executive function critical for stopping unwanted behavioral responses, impacts memory encoding (Chiu \& Egner, 2015a). In that study, participants performed a go/nogo task in which they were instructed to respond with a button press to one stimulus type (go) and to inhibit prepared motor responses to another stimulus type (no-go). Specifically, participants were instructed to execute motor responses to faces of one gender but to inhibit motor responses to faces of the other gender. In a subsequent surprise memory task, recognition memory scores for no-go stimuli were lower than recognition memory scores for go stimuli.

To explain these results, Chiu and Egner (2015a) proposed that attentional processes responsible for encoding the stimuli into memory competed with cognitive processes responsible 
for inhibiting the motor system. Because no-go stimuli are associated with recruiting the response inhibition system, the authors concluded that attentional resources had been diverted away from memory encoding, an effect termed inhibition-induced forgetting. A follow-up functional magnetic resonance imaging (fMRI) study examined the neural systems that underlie the inhibition-induced forgetting effect (Chiu \& Egner, 2015b). The authors found evidence to suggest that response inhibition and memory encoding processes competed for common neural resources: with increases in activation in a brain region associated with the deployment of inhibitory control (superior frontal gyrus), fewer resources were available that would otherwise serve to attend to and encode no-go stimuli into memory (ventrolateral prefrontal cortex). Thus, the inhibition-induced forgetting effect was explained in terms of response inhibition drawing neural resources away from memory encoding.

Alternatively, research has proposed an action-induced memory enhancement (Yebra et al., 2019) account to explain the pattern of go/no-go task memory scores. From this perspective, differences in recognition memory for go and no-go stimuli result from simple actions (e.g., button press) enhancing memory. Yebra et al. (2019) used converging fMRI and pupillometry data to support that overt action improves memory encoding. Specifically, the authors suggested that action execution engaged the locus coeruleus, which released noradrenaline, a neural resource known for improving memory encoding (Strange \& Dolan, 2004). The authors then examined the inhibition-induced forgetting prediction that greater inhibitory demands would result in poorer memory. It was reasoned that if inhibitory demands drive changes in no-go memory scores, then no-go stimuli that have a greater number of preceding go trials (e.g., three compared to one) should result in worse memory due to an increased prepotent tendency to respond (Thomas, Gonsalvez, \& Johnstone, 2009). Across a series of experiments, memory scores for no-go stimuli were not impacted by fluctuations in inhibitory demand. The effect of inhibitory demand on memory was also examined by manipulating the onset synchrony between stimulus encoding and response-inhibition processes. Yebra et al. (2019) proposed that if inhibitory demands impair memory encoding, then the effect should be greater when stimulus encoding coincides with the time point commonly associated with no-go trials' event-related potential peak amplitude (Kiefer, Marzinzik, Weisbrod, Scherg, \& Spitzer, 1998). Instead, response inhibition and stimulus encoding timing synchrony did not predict changes in memory scores. These findings provide evidence that fluctuations in inhibitory demand do not modulate memory encoding. Instead, the authors argued that memory encoding is influenced by action execution.

The line of work by Yebra et al. (2019) highlights the impact of overt motor responses on recognition memory and suggests that inhibitory demands do not directly impact the strength of memory encoding. What remains unclear, however, is how recognition memory for stimuli paired with a motoractive task, such as the go/no-go task, compare to stimuli that are paired with a motor-neutral task. Although previous research has focused on the inhibitory aspect of no-go trials, these trials also have a motor preparation component. Specifically, it is possible that during the go/no-go task, participants also prepare a response ahead of the presentation of a stimulus. A recent literature review of single pulse transcranial magnetic stimulation go/no-go studies (Ficarella \& Battelli, 2019) suggests that this assumption is dependent on several design factors, including (1) the probability of go and no-go stimuli (Fujiyama et al., 2012), (2) the time interval between a warning cue and stimulus onset (i.e., the foreperiod; Lebon et al., 2015; Touge et al., 1998), and (3) rewarding accurate responses (Freeman et al., 2014; Freeman \& Aron, 2016). The go/no-go task procedures used by Chiu and Egner (2015a, 2015b) and Yebra et al. (2019) were similar in that they both used an equiprobable distribution of go and no-go stimuli but also carried some notable differences. First, Chiu and Egner (2015a, 2015b) used very short foreperiods of 250 and 300 $\mathrm{ms}$, which are expected to increase motor preparation processes before trial onset (Tandonnet et al., 2010). Yebra et al. (2019, Experiment 2) only used a foreperiod in one of their experiments. However, in that experiment the authors still found a go versus no-go memory difference with (250 and $500 \mathrm{~ms}$ ) and without a foreperiod, in addition to finding similar reaction times on go trials across those foreperiods. Yebra et al. (2019, Experiment 3) also found that reward anticipation did not significantly alter the go versus no-go memory difference. Finally, we note that Chiu and Egner (2015a, Experiment 3) employed a stop-signal task, in which motor preparation processes are more reliably elicited due to a delay presented between the go and stop stimulus (Logan \& Cowan, 1984). The results of that study replicated the authors' other Experiments that used a go/no-go task. Together, these findings suggest that the different degrees to which motor processes are engaged between the no-go (motor preparation) and the go (motor execution) condition may instead explain how these stimuli are differentially encoded into memory.

Research using the go/no-go task paradigm has not yet systematically investigated the relative contribution of action preparation, inhibition, and execution on memory. Doing so requires comparing recognition memory of go and no-go stimuli to a condition that is absent of these motor processes but for which processing of the stimulus is still comparably task-relevant. It could be that no-go memory scores are weaker compared to a motor-neutral baseline, consistent with the inhibition induced-forgetting account. On the other hand, it could be that preparing a motor response on no-go trials enhances memory encoding relative to a motor-neutral baseline. We term this possibility the motor-induced encoding account to 
reflect that overt action is not required to improve memory encoding; rather, memory can be improved relative to the degree of motor engagement.

Other research supports the notion that motor preparation can impact cognitive processes. For example, it has been demonstrated that preparing a saccade or reaching behavior facilitates spatial attention to the goal location of the prepared movement (Baldauf, Wolf, \& Deubel, 2006; Baldauf \& Deubel, 2008; Hoffman \& Subramaniam, 1995; Shepherd, Findlay, \& Hockey, 1986). Enhanced discrimination of visual targets at the goal location of prepared movements has also been illustrated (Schneider \& Deubel, 1995). Furthermore, motor preparation has been shown to facilitate stimulus processing (Bosco, Daniele, \& Fattori, 2017; Gutteling, Kenemans, \& Neggers, 2011) for features that are relevant to the planned action, suggesting that motor preparation processes influence perceptual processes. Interestingly, some research suggests that action preparation, in addition to action execution, may engage the locus coeruleus (Naegeli et al., 2018; Pavlenko \& Kulichenko, 2003; Vitrac \& BenoiMarand, 2017). From this perspective, differences in memory recognition for go and no-go stimuli may instead result from differences in motor engagement. That is, go and no-go stimuli are both associated with the preparation of a motor response, but only go stimuli are associated with the execution of a prepared motor response. To our knowledge, research has not investigated the effect of motor preparation on memory or cognitive processes for stimuli that are unrelated to motor activity.

\section{Summary}

As this review indicates, several lines of work have applied different methods to determine how motor processes impact memory encoding. However, one aspect of memory recognition associated with go/no-go task performance that has not yet been explored is a comparison with a recognition baseline. Although differences in memory recognition have been observed between go and no-go stimuli, it is still unclear how these recognition scores compare to stimuli encountered in the absence of motor engagement. In the present study, we replicated and extended upon previous literature by directly examining how memory recognition scores for motor engagement conditions (i.e., action preparation, inhibition, and execution) would compare to a motor-neutral condition. According to the inhibition-induced forgetting account, competition arising between response inhibition and stimulus encoding processes should impair memory for no-go stimuli compared to go stimuli and motor-neutral stimuli. According to the action-induced memory enhancement account, an inverse process should occur by which overt action in the go condition should enhance memory scores beyond motor-neutral stimuli and no-go stimuli. Alternatively, it could be that both go stimuli and no-go stimuli are remembered better than motor-neutral stimuli due to their engagement of the motor system, consistent with the motor-induced encoding account.

\section{Experiment 1}

The purpose of Experiment 1 was to examine memory ratings associated with action execution and the inhibition of prepared actions. Here, we replicated the procedure of Chiu and Egner (2015a, 2015b) first to establish that we could observe the same pattern of results showing that memory recognition for stimuli accompanied with action execution is superior to that of stimuli accompanied with prepared but inhibited actions. The experiment consisted of three sequential phases, as illustrated in Fig. 1. First, participants performed the go/no-go task. Next, a filler task was administered which served only as an encoding-retrieval delay period. Lastly, participants performed a surprise recognition memory task in which faces were presented from the go/no-go task intermixed with novel face stimuli. Participants reported on a confidence scale if they had seen the face before or if it was new. All subsequent experiments followed this same sequence: first, participants were administered a task that exposed them to a set of face stimuli, followed by an encoding-retrieval delay task, and finally a surprise recognition memory task.

\section{Methods}

Participants Thirty-three undergraduate students (19 female) aged 18-30 years from the University of Tennessee, Knoxville, participated. We determined our sample size based on the results of Chiu and Egner (2015b). Because we had a priori planned to implement a third experimental condition for subsequent experiments, we performed power calculations for a design composed of one factor with three levels. With a desire for high power (0.9), we determined a target sample of 32 participants based on the go versus no-go memory effect. We used the same sample size for all subsequent experiments. Participants were compensated with class credit upon completion. Participants completed an informed consent and demographics survey upon arrival. The Institutional Review Board approved the study's protocol. One participant was excluded for hit rates in the memory task falling more than two standard deviations below the group mean.

Stimuli Two hundred and forty face stimuli (half male/female) were collected from the Productive Aging Laboratory Face Database (Minear \& Park, 2004), the Cohn-Kanade Facial Expression DataBase (Kanade et al., 2000), and the Chicago Face Database (Ma, Correll \& Wittenbrink, 2015). All stimuli were grayscale and affectively neutral. We randomly distributed stimuli into four sets of 60 stimuli each (gender equated). 
a

\section{Phase 1}

Experiment 1:

Go/no-go task

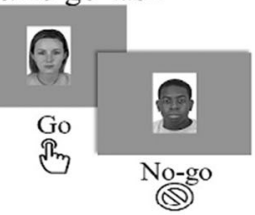

b Experiment 2a:

(iQ)

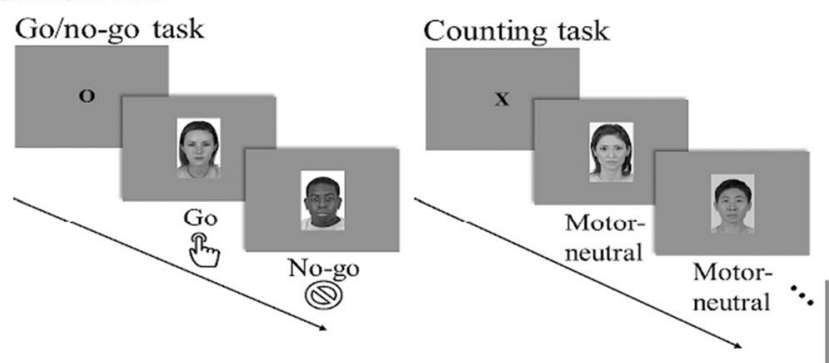

C

\section{Experiment 2b:}

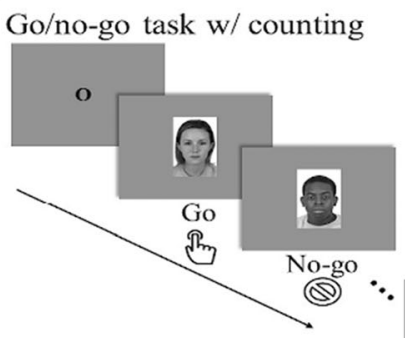

Counting task

d

\section{Experiment 3:}

Go/no-go task
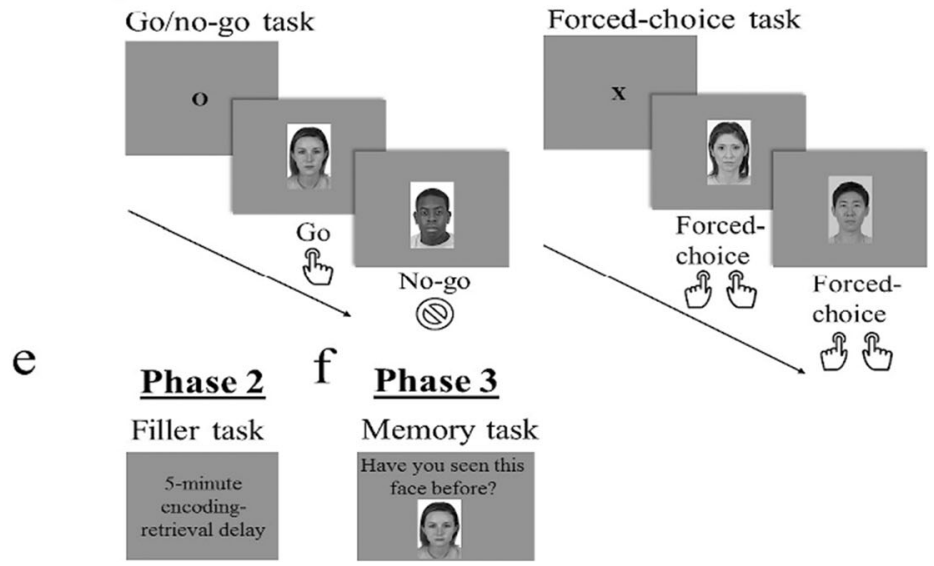

Fig. 1 Task paradigms across experiments. In phase 1 for each experiment, participants were exposed to a set of face stimuli. (a) In Experiment 1, participants identified face stimuli as female or male by executing or suppressing a motor response in a go/no-go task. (b) In Experiment 2a, the letter "O" or " $\mathrm{X}$ " cued participants to engage in either the go/no-go task or the counting task. In the counting task, participants counted and then reported the number of male and female faces (motor-neutral) that were presented. (c) In Experiment $2 b$, participants continuously counted and then reported the number of male

Two stimulus sets were used in the go/no-go task and were presented in the go or no-go condition an equal number of times across participants. Two stimulus sets only appeared

and females faces that were presented in each section. The counting task either coincided with the go/no-go task or occurred by itself (as in Experiment 2a). (d) In Experiment 3, participants engaged in go/no-go responses or identified male and female stimuli by executing forcedchoice responses. (e) After phase 1 of each experiment, a filler task was administered to create a 5-min encoding-retrieval delay period. (f) Lastly, in phase 3 participants' incidental recognition memory was measured using a surprise memory task that incorporated "old" stimuli from phase 1 and a set of "new" stimuli.

in the memory-recognition task, which stayed constant across participants. The filler task included 78 grayscale stimuli (39 house/apartment stimuli). This stimulus set size was chosen to 
fulfill a 5-min encoding-retrieval delay period before the surprise memory task.

\section{Design and procedure}

Go/no-go task Participants were instructed to respond using the spacebar to one gender (go) of face stimuli and to withhold a response to the other gender (no-go; see Fig. 1a). Each trial began with a fixation cross $(250 \mathrm{~ms})$, followed by the presentation of a face stimulus $(800 \mathrm{~ms})$. Thus, there was a foreperiod of $250 \mathrm{~ms}$ to encourage motor preparation processes before trial onset (Davanche et al., 2007; Tandonnet et al., 2010). On go trials, participants had until stimulus offset to execute a response. An equal number of inter-trial intervals were randomly distributed across trials and were jittered from $2.5 \mathrm{~s}$ to $4.5 \mathrm{~s}$ (step sizes of $250 \mathrm{~ms}$ ). Four blocks of 120 trials each were administered in the go/no-go task, with each face stimulus appearing once per block, thus creating 480 total trials.

Filler task The Filler task acted as an encoding-retrieval delay period ( $5 \mathrm{~min}$; see Fig. 1e) before the surprise recognition memory task. Participants were presented with house and apartment stimuli and were tasked to categorize them using the $\mathrm{z}$ and $\mathrm{m}$ keys. Participants completed one block of 78 trials. Presentation timings were the same as those used in the go/no-go task except for inter-trial intervals, which were set at $2 \mathrm{~s}$.

Memory task Incidental recognition memory was then tested for the face stimuli presented in the go/no-go task (see Fig. 1f). To do this, we included all 120 face stimuli from the go/no-go task ("old" stimuli) and added 120 face stimuli ("new" stimuli), thus creating 240 total trials. The presentation of "new" and "old" stimuli was randomized. At the start of each trial, participants were shown a face stimulus for $2 \mathrm{~s}$ and then a 6point scale, which ranged from "definitely new" to "definitely remember." The scale remained on the screen until a response with the mouse was executed. Inter-trial intervals were equally distributed across trials and were jittered from $2.5 \mathrm{~s}$ to $4.5 \mathrm{~s}$ (step sizes of $250 \mathrm{~ms}$ ).

\section{Results}

Go/no-go task As expected, overall accuracy was high ( $M=$ 98.3\%, SEM $=0.002$; see Table 1), suggesting that participants performed the task as instructed. Accuracy on go trials $(M=98.1 \%, S E M=0.003)$ did not significantly differ from accuracy on no-go trials $(M=98.5 \%, S E M=0.001), t(31)=-$ $1.22, p=.23, d=0.44$

Memory task We first tested if memory recognition varied as a function of the gender of faces presented in the go/no-go task and the gender of participants. We found no difference in memory recognition between stimuli that had the same $(M=$ 1.55$, SEM $=.15)$ or different $(M=1.44, S E M=.13)$ gender as participants, $t(31)=1.64, p=.11, d=0.59$. For subsequent analyses, we simply collapsed across stimulus and participant genders. Shifting to our primary area of interest, we measured the effect of go and no-go responses on memory recognition. As in Chiu and Egner (2015a, 2015b), only correct stimuli (i.e., action correctly executed or inhibited for all four stimulus repetitions) from the go/no-go task were considered in the memory analyses. Memory-task responses that exceeded $2 \mathrm{~s}$ were also excluded. We grouped "definitely new" to "maybe new" responses into the "new" category and "definitely remember" to "maybe remember" responses into the "remember" category. Follow-up experiments used these same datapreprocessing steps. A one-sample t-test revealed that overall hit rates $(M=72.8 \%, S E M=0.03)$ were above chance level, $t(31)=9.22, p<.001, d=3.31$. We then calculated $d^{\prime}$ (z(hit rate) $-z$ (false-alarm rate) $)$ as a measure of memory recognition strength for go and no-go stimuli (see Fig. 2a). We found significantly better memory scores for go stimuli $\left(d^{\prime}=1.59\right.$, $S E M=0.15)$ compared to no-go stimuli $\left(d^{\prime}=1.41, S E M=\right.$ 0.14 ; see Table 2), $t(31)=2.81, p=.008, d=1.03$.

\section{Discussion}

Our results replicated previous findings such that no-go stimuli resulted in worse recognition memory than go stimuli (Chiu \& Egner, 2015a; Yebra et al., 2019). Next, we examined whether coupling memory encoding processes with action (go) facilitates memory encoding, if the recruitment of an inhibitory system (no-go) impairs memory encoding, or if motor-engagement processes underlying both of these conditions enhance memory encoding. We tested this by incorporating a motor-neutral condition that still required participants to encode the gender of face stimuli, but such that the preparation, inhibition, or execution of a motor response was not required.

\section{Experiment 2a}

In Experiment 2a, we added a condition composed of motorneutral stimuli that were not associated with the engagement of the motor system. If response inhibition reduces the availability of resources for memory encoding, as proposed by the inhibition-induced forgetting account, then we would expect motor-neutral stimuli to show better recognition compared to no-go stimuli. Alternatively, the action-induced memory enhancement account predicts that only memory scores for stimuli associated with action should be enhanced, such that go stimuli, but not no-go stimuli, would result in better recognition memory compared to motor-neutral stimuli. Finally, the motor-induced encoding account predicts that memory scores 
Table 1. Average accuracy per condition across experiments

\begin{tabular}{lllll}
\hline Experiment & Go & No-go & $\begin{array}{l}\text { Motor- } \\
\text { neutral } \\
M(\text { SEM })\end{array}$ & $\begin{array}{l}\text { Forced- } \\
\text { choice } \\
M(\text { (SEM) }\end{array}$ \\
\hline 1 & $M($ SEM) & $M(S E M)$ & $98.6 \%(.003)$ \\
$2 \mathrm{a}$ & $98.1 \%(.003)$ & $98.5 \%(.001)$ & $98.3 \%(.012)$ \\
$2 \mathrm{~b}$ & $96.3 \%(.006)$ & $98.9 \%(.002)$ & $93.0 \%(.01)$ \\
3 & $91.0 \%(.011)$ & $99.3 \%(.002)$ & \\
\hline
\end{tabular}

for stimuli associated with motor engagement (go and no-go) should be enhanced compared to motor-neutral stimuli. To test these accounts, we implemented three conditions of varying motor demands: motor-neutral (absent of motor processes), no-go (motor preparation and inhibition), and go (motor preparation and execution). In alternating blocks, participants either performed the original go/no-go task or a counting task. We decided to alternate these tasks so that the delay between exposure and recognition testing would not be confounded

\section{a}

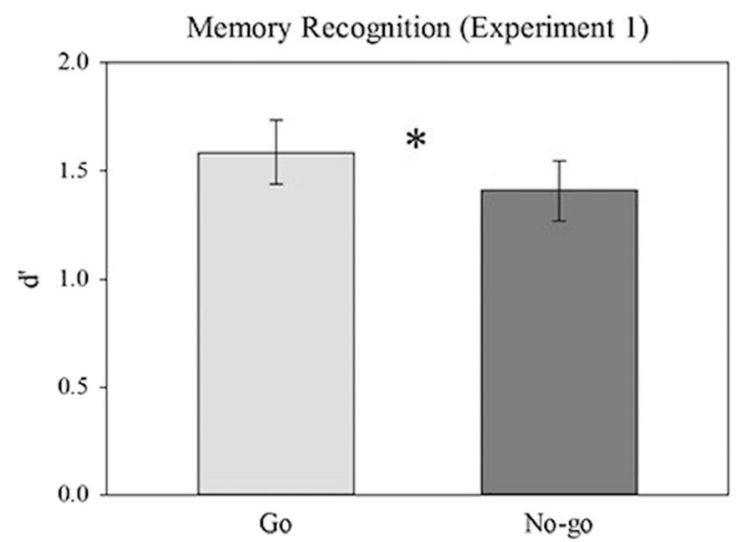

$\mathrm{C}$

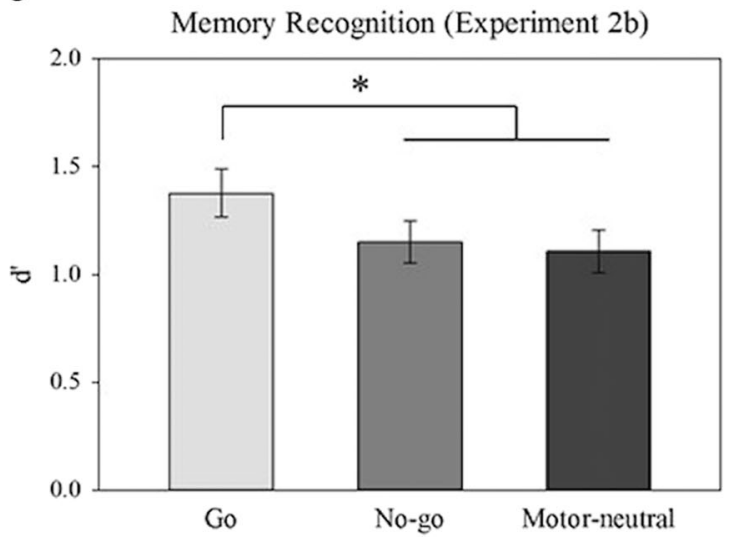

Fig. 2 Memory recognition results ( $d$ ') across experiments. (a) Memory recognition scores for go stimuli were superior to no-go stimuli. (b) Memory recognition scores for motor-neutral stimuli were inferior to that of go and no-go stimuli. (c) Memory recognition scores for go stimuli were higher than no-go stimuli and motor-neutral stimuli. Memory with condition. During the counting task, participants were presented with a series of face stimuli and counted the number of male and female faces that were shown. At the end of each counting-task section, participants reported the number of faces there was of each gender. Although the counting task elicits subvocal responses (counting), this form of response is uncoupled from a motor response system and would be distinct from those processes engaged by the go/no-go task. Thus, the counting task provides a motor-neutral condition

b

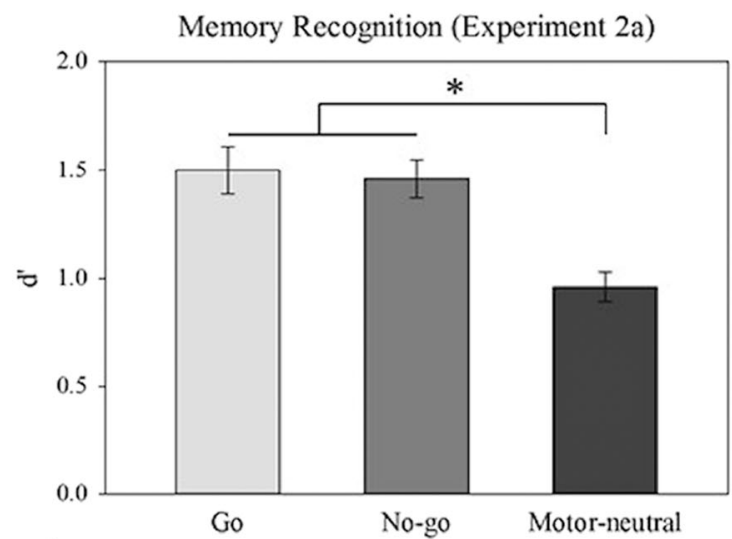

d

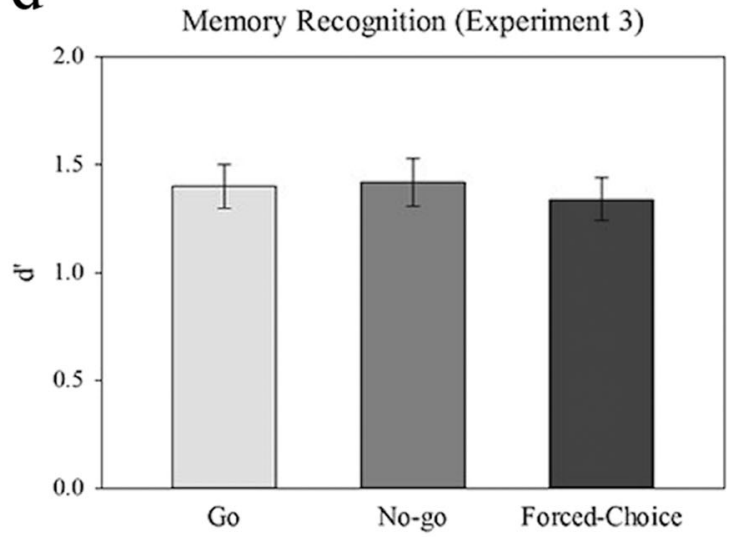

recognition scores for no-go stimuli and motor-neutral stimuli did not significantly differ. (d) Memory recognition scores did not significantly differ between the go, no-go, and forced-choice conditions. Error bars display SEM. $* p<.05$ 
Table 2. Average memory recognition (d') per condition across experiments

\begin{tabular}{lllll}
\hline Experiment & Go & No-go & $\begin{array}{l}\text { Motor- } \\
\text { neutral } \\
M(S E M)\end{array}$ & $\begin{array}{l}\text { Forced- } \\
\text { choice } \\
M(S E M)\end{array}$ \\
\hline 1 & $M(S E M)$ & $M(S E M)$ & & \\
$2 \mathrm{a}$ & $1.59(.15)$ & $1.41(.14)$ & & \\
$2 \mathrm{~b}$ & $1.30(.10)$ & $1.25(.09)$ & $1.01(.07)$ & \\
3 & $1.37(.11)$ & $1.14(.09)$ & $1.10(.09)$ & $1.34(.10)$ \\
\hline
\end{tabular}

that requires comparable stimulus processing steps to the go/ no-go task (i.e., participants were required to process the stimulus to discriminate whether it was male or female) with the exception that it did not engage the motor system. The go/nogo task used here was identical to Experiment 1, with the exception that there were fewer trials per block to accommodate the alternating task-structure.

\section{Methods}

Participants Thirty-three undergraduate students (17 female) aged 18-30 years were recruited for participation. Participants completed an informed consent and demographics survey upon arrival, approved by the Institutional Review Board. One participant was excluded due to hit rates falling more than two standard deviations below the group mean.

Stimuli The same array of 240 stimuli were used from Experiment 1. To incorporate a third experimental condition, we generated four new sets of unique stimuli. Stimuli were distributed into these sets based on their average memory ratings from Experiment 1, collapsed across the go and no-go conditions. More specifically, stimuli were divided into sets in alternating order from highest to lowest average memory ratings. Of these four new sets of stimuli, two contained 30 male faces each and two contained 30 female faces each. The same four stimulus sets were used across participants, with each set being featured in the go condition, no-go condition, or motorneutral condition equally. Participants were assigned one set to the go condition and one set to the no-go condition. For the counting task, 30 total stimuli were used in which 15 stimuli were randomly selected from each of the remaining two sets of stimuli. Thus, each participant saw 30 stimuli in the go condition, 30 stimuli in the no-go condition, and 30 stimuli in the counting task (15 male/female). Because fewer stimuli were now presented in the first phase of tasks, participants saw three repetitions for each face instead of four. We used fewer stimulus repetitions to maintain the same repetition to total stimuli ratio from Experiment 1 and to avoid a memory ceiling effect. As in Experiment 1, another set of 120 stimuli was only used for the memory task that served as "new" stimuli. To match the number of stimuli from the first phase of tasks, a total of 90 "new" stimuli (45 male/female) were randomly selected to be shown in the memory-recognition task.

\section{Design and procedure}

The basic structure of the tasks is shown in Fig. 1. In alternating sections, participants either performed the go/no-go task or the counting task (see Fig. 1b). A letter cue of either an $\mathrm{O}$ or $\mathrm{X}$ signaled and preceded the go/no-go and counting sections, respectively. The sequencing of tasks was randomly determined across participants. Participants completed a total of 18 sections (nine of each task) with 90 total stimuli presented across these sections. Three blocks were administered that consisted of six sections (three of each task) per block. Participants saw each face stimulus three times across these blocks (once per block), thus creating a total of 270 trials. The filler task and memory task was identical to that of Experiment 1.

Counting task Participants were instructed to count the number of male and female faces presented in each section (motorneutral). Importantly, participants were given explicit instructions to count the stimuli silently and internally (i.e., avoid counting using fingers or speech). Thus, motor-neutral stimuli were void of motor processing. Thirty stimuli (15 male/female) were used in the counting task. Ten stimuli were presented in each section. Presentation timings were the same as those used in the go/no-go task. Stimuli consisted of male and female combinations of six and four, five and five, or four and six, respectively. Each combination was used three times throughout the task (once per block). At the end of each section, two scales ranging from three to seven were presented asking "How many female faces?" and "How many male faces?" Participants used the mouse to respond, and the scale remained on the screen until a response was recorded. The order of scale presentation (male or female test) was randomized for each section.

Go/no-go task Sixty stimuli were used in the go/no-go task. Twenty stimuli (ten go and ten no-go) were presented in each section. The go/no-go task was otherwise identical to that of Experiment 1.

\section{Results}

Go/no-go task Accuracy for go and no-go stimuli was high ( $M$ $=97.6 \%, S E M=0.005$; see Table 1), and performance was significantly better for no-go stimuli $(M=98.9 \%, S E M=$ $0.002)$ than go stimuli $(M=96.3 \%, S E M=0.006), t(31)=$ $4.0, p<.001, d=1.46$.

Counting task Overall accuracy was high for reporting the number of male and female stimuli that were presented in each 
section $(M=98.6 \%, S E M=0.003$; see Table 1$)$. There was no difference in accuracy for reports between male $(M=98.6 \%$, $S E M=0.004)$ and female stimuli $(M=98.7, S E M=0.003)$, $t(31)=-.38, p=.70, d=0.14$.

Memory task Overall hit rates $(M=67.5 \%, S E M=0.02)$ were significantly above chance level, $t(31)=8.06, p<.001, d=$ 2.94. The $d$ ' scores for this experiment are shown in Fig. 2b. A one-factor one-way ANOVA with three conditions (go, nogo, and motor-neutral) revealed that there was a significant main effect of condition on recognition (see Table 2), $F(2,62)=7.79, p=.001, \eta_{\mathrm{p}}^{2}=.20$. Follow-up Bonferronicorrected pair-wise comparisons revealed no difference in memory scores between go $\left(d^{\prime}=1.30, S E M=0.10\right)$ and nogo stimuli $\left(d^{\prime}=1.25, S E M=0.09\right), p>.9$. However, memory score for motor-neutral stimuli $\left(d^{\prime}=1.01, S E M=.07\right)$ was inferior to that of go and no-go stimuli ( $p=.001$ and $p=.01$, respectively). We also found a significant linear contrast, indicating that memory scores increased across levels of the motor-neutral, no-go, and go conditions, $F(1,31)=15.94, p$ $<.001, \eta_{\mathrm{p}}^{2}=.34$.

\section{Discussion}

The present experiment tested the influence of motor processes on memory encoding. To do this, we included a motorneutral condition as a baseline comparison group. Our results showed that the go and no-go conditions had enhanced memory scores compared to the motor-neutral condition, providing the first evidence for motor engagement improving memory as predicted by the motor-induced encoding account. One alternative explanation for the pattern of memory scores observed in Experiment 2a is the addition of working memory demands (i.e., actively maintaining the number faces throughout each section) in the counting task but not in the go/no-go task. That is, working memory demands during the counting task may have worsened memory encoding for motor-neutral stimuli, as opposed to a lack of motor system engagement. The following experiment aimed to control for this possible confound by adding working memory demands to the go/nogo task to further examine the effect of motor processes on memory.

\section{Experiment 2b}

Experiment $2 \mathrm{a}$ found that go and no-go stimuli had better memory scores than motor-neutral stimuli. However, memory encoding for motor-neutral stimuli could have been worse than go stimuli and no-go stimuli due to a non-motor factor. Specifically, the working memory demands imposed by counting and remembering the gender of motor-neutral stimuli may have impaired memory encoding relative to go stimuli and no-go stimuli. Previously, we aimed to keep the processes involved in the go/no-go task from Experiment 1 and Experiment $2 \mathrm{a}$ consistent. In the present Experiment, however, we modified the go/no-go task to control for working memory effects. In this way, go/no-go stimuli included characteristics (i.e., counting/working memory demands) of motor-neutral stimuli while still engaging the motor system. These changes also allowed for processes involved in counting the stimuli to remain constant between both tasks.

\section{Methods}

Participants Thirty-four undergraduate students (22 female) aged 18-30 years were recruited for participation. Participants completed an informed consent and demographics survey upon arrival, approved by the Institutional Review Board. One participant was excluded for not following task instructions (did not respond to go stimuli), and one participant was excluded for hit rates falling more than two standard deviations below the group mean.

Stimuli Stimulus sets were the same as those used in Experiment 2a.

\section{Design and procedure}

The design and procedure of Experiment $2 b$ were identical to Experiment 2a, except that participants also counted the number of face stimuli (male and female) while preparing then inhibiting or executing motor responses in each go/no-go task section (ten stimuli per section; see Fig. 1c).

\section{Results}

Go/no-go task with counting Overall response accuracy was high $(M=95.1 \%, S E M=0.01$; see Table 1$)$, and accuracy for go stimuli $(M=91.0 \%, S E M=0.01)$ was significantly lower than no-go stimuli $(M=99.3 \%, S E M=0.002), t(31)=-7.05, p$ $<.001, d=2.57$. Counting accuracy was also high for go and no-go stimuli $(M=99.1 \%, S E M=0.001)$.

Counting task Response accuracy in the counting task ( $M=$ $98.3 \%, S E M=.01$; see Table 1) indicated that participants performed the task as instructed. Counting accuracy for motor-neutral stimuli $(M=99.3 \%, S E M=.001)$ did not differ from counting accuracy for go/no-go stimuli, $t(31)=-.745, p$ $=.462, d=0.27$.

Memory task Overall hit rates $(M=65.9 \%, S E M=0.02)$ were significantly above chance level, $t(31)=6.42, p<.001, d=$ 2.34. The $d$ ' scores for this experiment are shown in Fig. 2c. There was a main effect of condition on recognition (see Table 2), $F(2,62)=7.29, p=.002, \eta_{p}^{2}=.191$. Memory 
recognition scores were significantly higher for go stimuli ( $d$ ' $=1.37, S E M=0.11)$ than no-go stimuli $\left(d^{\prime}=1.14, S E M=\right.$ $0.09 ; p=.03)$ and motor-neutral stimuli $\left(d^{\prime}=1.10, S E M=\right.$ $0.09 ; p=.006)$. Memory recognition scores between no-go stimuli and motor-neutral stimuli did not differ, $p>.9$. However, replicating Experiment 2a, we found a significant linear contrast, such that memory scores increased across levels of the motor-neutral, no-go, and go conditions, $F(1,31)=11.19, p=.002, \eta_{\mathrm{p}}^{2}=.26$.

Between-experiment comparisons Next we performed a 2 (condition; go, no-go) $\times 2$ (counting; present, absent) mixed ANOVA with condition as a within-subjects factor and counting as a between-subjects factor across Experiment $2 \mathrm{a}$ and Experiment $2 b$ to test whether working memory demands impacted memory encoding. Go stimuli $\left(d^{\prime}=1.34\right.$, SEM $=$ $.07)$ had significantly higher recognition scores than no-go stimuli $\left(d^{\prime}=1.20, S E M=.06\right), F(1,62)=5.29, p=.02, \eta_{\mathrm{p}}^{2}$ $=.079$. There was no difference in memory recognition scores when counting was absent $\left(d^{\prime}=1.28, S E M=.09\right)$ or present $\left(d^{\prime}=1.26, S E M=.09\right), F(1,62)=0.016, p=.89, \eta_{\mathrm{p}}^{2}=.0002$, and there was no interaction of condition and counting on memory scores, $F(1,62)=2.32, p=.13, \eta_{\mathrm{p}}^{2}=.036$.

To examine patterns of memory scores between conditions across Experiments $2 \mathrm{a}$ and $2 \mathrm{~b}$, we performed a 3 (condition; go, no-go, motor-neutral) $\times 2$ (experiment: $2 \mathrm{a}, 2 \mathrm{~b})$ mixed ANOVA with condition as a within-subjects factor and experiment as a between-subjects factor. This comparison only revealed a main effect of condition, $F(2,124)=13.08, p<.001$. Follow-up comparisons showed that go $\left(d^{\prime}=1.34, S E M=\right.$ .08 ) and no-go stimuli ( $d^{\prime}=1.20$, SEM $\left.=.07\right)$ had significantly higher memory scores than motor-neutral stimuli $\left(d^{\prime}=1.06\right.$, $S E M=.06 ; p<.001$ and $p=.017$, respectively). Go stimuli also had higher memory scores than no-go stimuli, but this effect was only marginally significant, $p=.07$.

\section{Discussion}

Experiment $2 \mathrm{~b}$ further examined the effect of motor processes on memory encoding by controlling for working memory demands. Our results replicated Experiment 2a, such that go stimuli had higher memory scores than motor-neutral stimuli. Additionally, go stimuli had higher memory scores than no-go stimuli, replicating the results of Experiment 1. Unlike Experiment 2a, memory scores for no-go stimuli were not different from motor-neutral stimuli. It is possible that because no-go stimuli have weaker motor engagement compared to go stimuli, introducing working memory demands to these stimuli divided attention, subsequently diminishing the memory benefit that was observed in Experiment 2a. Importantly, however, when we compared memory performance across Experiments $2 \mathrm{a}$ and $2 \mathrm{~b}$, we found that both go and no-go stimuli had better memory scores than motor-neutral stimuli.
Furthermore, we found no difference in go/no-go task memory scores between Experiments $2 \mathrm{a}$ and $2 \mathrm{~b}$. This result suggests that the additional working memory demands in the counting task cannot account for the reduced memory scores in the motor-neutral condition. Instead, we suggest that memory performance for motor-neutral stimuli was lower due to the absence of motor engagement. Our results support a motor-induced encoding effect, in which memory scores were boosted for stimuli that were accompanied by action preparation or action execution. In contrast to the inhibition-induced forgetting account, the pattern of memory scores is better explained by the degree of motor engagement rather than response inhibition. Further, in contrast to the action-induced memory enhancement account, we found evidence that action execution was not required to enhance recognition memory.

\section{Experiment 3}

Across Experiments $2 \mathrm{a}$ and $2 \mathrm{~b}$, we found that go and no-go stimuli had better memory scores than motor-neutral stimuli. We suggest that these results arose from the lack of motor system engagement for motor-neutral stimuli. To further explore the relative contribution of the motor system to memory encoding, Experiment 3 replicated the procedure from Experiment 2a but modified the sections of trials in which participants were performing the counting task to instead engage the motor system. Specifically, participants were now required to execute a forced-choice motor response to indicate whether faces during these sections were either male or female. This experiment will also allow us to examine the influence of task-switching on recognition memory. Specifically, from the perspective of the motor-induced encoding account, recognition memory scores should track the degree of motor engagement. Thus, we expected that stimuli in the forced-choice condition would now yield memory scores comparable to those for go and no-go stimuli and higher than motor-neutral stimuli from Experiment 2a. However, if recognition memory scores for these stimuli are still lower than go and no-go stimuli, this would suggest that the imbalance in recognition previously reported between go, nogo and motor-neutral stimuli may be attributed to taskswitching from the go/no-go task to the counting task as opposed to a lack of motor system engagement.

\section{Methods}

Participants Thirty-four undergraduate students (22 female) aged 18-30 years were recruited for participation. Participants completed an informed consent and demographics survey upon arrival, approved by the Institutional Review Board. One participant was excluded for choosing not to finish the experiment, and one participant was excluded for disclosing prior knowledge that there would be a surprise memory task. 
Stimuli Stimulus sets were the same as those used in Experiment 2a and $2 b$.

\section{Design and procedure}

The design and procedure of Experiment 3 were the same as Experiment $2 \mathrm{a}$ with the exception that instead of the counting task, participants performed a forced-choice task (shown in Fig. 1d).

Forced-choice task Participants were instructed to press the $z$ key for one gender of faces and the $m$ key for the other gender. Half of the participants responded to male stimuli with the $\mathrm{z}$ key, while the other half responded to female stimuli with the $\mathrm{z}$ key.

\section{Results}

Go/no-go task Overall accuracy was high $(M=96.6 \%$, SEM = 0.008 ; see Table 1), and there was no difference in accuracy between go $(M=96.7 \%, S E M=0.01)$ and no-go stimuli $(M=$ $96.5 \%, S E M=0.006), t(31)=.20, p=.84, d=0.07$.

Forced-choice task Accuracy in the forced-choice task ( $M=$ $93 \%, S E M=.01$; see Table 1) indicated that participants performed the task as instructed, but with worse accuracy than that of the go/no-go task $(M=96.6 \%, S E M=0.008), t(31)=$ 3.78, $p=.001, d=1.38$. An independent-samples t-test showed that accuracy in the forced-choice task $(M=93 \%$, $S E M=.01)$ was worse than accuracy in the counting task $(M=98.6 \%, S E M=0.003)$ used in Experiment 2a, $t(62)=$ $3.94, p<.001, d=0.98$.

Memory task Hit rates $(M=69.6 \%, S E M=0.02)$ were significantly above chance level, $t(31)=9.08, p<.001, d=3.31$. The $d$ ' scores for this experiment are shown in Fig. 2d. There was no effect of condition on recognition (see Table 2), $F(2,62)=.60, p$ $=.55, \eta_{\mathrm{p}}^{2}=.018$; recognition between go $\left(d^{\prime}=1.40, S E M=\right.$ $0.10)$, no-go $\left(d^{\prime}=1.42, S E M=0.11\right)$, and forced-choice stimuli $\left(d^{\prime}=1.34, S E M=0.10\right)$ did not differ. A linear contrast to test whether memory scores increased across levels of condition was not significant, $F(1,31)=1.36, p=.25, \eta_{\mathrm{p}}^{2}=.04$.

Between-experiment comparisons An independent-samples t-test revealed that the forced-choice stimuli $\left(d^{\prime}=1.34\right.$, $S E M=0.10$ ) in Experiment 3 had significantly higher memory scores than the motor-neutral stimuli $\left(d^{\prime}=1.01\right.$, $S E M=0.07)$ used in the counting task in Experiment 2a, $t$ $(62)=2.63, p=.01, d=0.67$.

We then assessed whether go/no-go task accuracy varied across our Experiments. To do this, we submitted a 2 (condition: go, no-go) $\times 4$ (experiment: $1,2 \mathrm{a}, 2 \mathrm{~b}, 3)$ mixed ANOVA with condition as a within-subjects factor and experiment as a between-subjects factor. There was a significant main effect of condition on accuracy, $F(1,124)=$ $41.54, p<.001, \eta_{\mathrm{p}}^{2}=.251$; performance on go trials $(M=$ $95.6 \%, S E M=0.004)$ was significantly worse than no-go trials $(M=98.3 \%, S E M=0.002)$. There was a significant main effect of experiment on accuracy, $F(3,124)=6.8, p<$ $.001, \eta_{p}^{2}=.141$. Follow-up Bonferroni-corrected pair-wise comparisons revealed that go/no-go task accuracy in Experiment $2 \mathrm{~b}(M=95.2 \%, S E M=.005)$ was significantly worse compared to Experiment $1(M=98.3 \%, S E M=$ $.005 ; p<.001)$ and Experiment $2 \mathrm{a}(M=97.7 \%, S E M=$ $.005 ; p=.007)$. There was also a significant interaction of condition and experiment, $F(3,124)=20.49, p<.001, \eta^{2}$ $=.331$. For Experiments $2 \mathrm{a}$ and $2 \mathrm{~b}$, the no-go condition had higher accuracy than the go condition $(p=.003$ and $p$ $<.001$, respectively). For Experiments 1 and 3, there was no effect of condition on accuracy $(p=.67$ and $p=.81$, respectively).

Next, we examined whether task switching in Experiments $2 \mathrm{a}, 2 \mathrm{~b}$, and 3 impacted memory scores in the go/no-go task. We did this by comparing go/no-go memory scores in Experiment 1 (in which there was no task switching) to the go/no-go memory scores of Experiments 2a, 2b, and 3 that included task switching. Specifically, we performed a 2 (condition: go, no-go) $\times 4$ (experiment: $1,2 \mathrm{a}, 2 \mathrm{~b}, 3)$ mixed ANOVA with condition as a within-subjects factor and experiment as a between-subjects factor. First, this analysis showed a significant main effect of condition on memory scores, $F(1,124)=6.97, p=.009, \eta_{\mathrm{p}}^{2}=.053$. This analysis did not reveal a significant main effect of experiment on memory scores, $F(3,124)=1.03, p=.38, \eta_{p}^{2}=.024$, and there was not a significant interaction of condition and experiment, $F(3,124)=2.21, p=.09, \eta_{\mathrm{p}}^{2}=.053$. Finally, we note that the dependent measures of memory score and accuracy across experiments were not significantly associated on go trials, $r(126)=.085, p=.34$, or on no-go trials, $r(126)=.075, p=.4$.

\section{Discussion}

Experiment 3 further examined the effect of motor engagement on memory encoding. We used the same procedure as in Experiment 2a, with the exception that forced-choice stimuli replaced motor-neutral stimuli. Our results showed that the go, no-go, and forced-choice stimuli had similar memory scores. We also found that forced-choice stimuli had better memory recognition than motor-neutral stimuli from Experiment 2a, despite the forced-choice task having significantly worse accuracy than the counting task. These results suggest that the motor demands applied to forced-choice stimuli boosted recognition scores relative to the motor-neutral stimuli, supporting a motor-induced encoding effect in which memory scores fluctuated in the presence or absence of motor processes. 


\section{General discussion}

The present study examined the influence of motor processes on memory encoding. First, we replicated prior research, finding that stimuli paired with an action (go) were subsequently remembered better than stimuli prompting the inhibition of a prepared action (no-go; Experiment 1). We then asked if these go and no-go memory differences were the result of response inhibition impairment, overt action enhancement, or rather differences in the degree of motor engagement (i.e., motor preparation versus motor execution). To test this, Experiments $2 \mathrm{a}$ and $2 \mathrm{~b}$ included motor-neutral stimuli that did not engage the motor system. Across these experiments, our results revealed that stimuli associated with motor preparation and motor execution processes had significantly better memory scores than stimuli uncoupled with motor processes. Contrary to the inhibition-induced forgetting account, no-go stimuli were associated with superior memory recognition compared to motor-neutral stimuli. From these observations, we conclude that response inhibition does not impose an adverse role in memory encoding. Instead, these findings support a motor-induced encoding effect such that the preparation of motor responses can also improve memory encoding. This perspective goes beyond the action-induced memory enhancement account by proposing that motor processing, including both action execution and action preparation, enhance memory scores. The motor-induced encoding account was further supported by Experiment 3, which found that by introducing motor demands in the form of forced-choice responses, memory recognition was boosted compared to the motor-neutral stimuli in Experiment 2a.

Interestingly, we did not find memory recognition differences between the go and no-go conditions in all of our experiments (see Table 3 for a breakdown of memory effects). In line with previous research, the go versus no-go memory effect appears subtle (Chiu \& Egner 2015a, 2015b). For example, Yebra et al. (2019, Experiments 5, 7a, and 7b) also found null effects for the go versus no-go comparison of memory scores in some experiments; however, a meta-analysis revealed a go versus no-go memory effect across all of their experiments. Similarly, we also found that go stimuli were remembered better than no-go stimuli when collapsed across all of our experiments. It could be that observing a memory difference between go and no-go stimuli depends on taskrelated factors that modulate motor demands. As discussed previously, research suggests that design characteristics of the go/no-go task (e.g., the foreperiod and go/no-go trial ratio) elicit fluctuations in motor preparation. While the difference in motor processing between go and no-go stimuli was intended to remain constant across our experiments, it is possible that manipulations to the task design altered go or no-go motor processes. For example, task switching in Experiments $2 \mathrm{a}$ and 3 may have led to greater motor engagement on no-go trials, thus resulting in similar memory scores compared to go stimuli. However, in an analysis between those Experiments, we found that task switching did not significantly alter go and no-go memory encoding. While Experiment $2 b$ also included task switching and we found a go versus no-go memory difference, the presence of working memory demands in the go/ no-go task may have diverted attention away from stimulus encoding on no-go trials, where there is weak coupling to motor processes compared to go trials. The variation in go and no-go memory scores may also suggest that there is some common contribution to memory encoding that results from task manipulations to motor preparation processes. On that basis, the motor-induced encoding account offers an explanation for the different findings between go and no-go memory scores.

Although open questions remain in this line of work, the most important finding in our results showed that stimuli coupled with motor engagement processes (go, no-go, and forced-choice) reliably had enhanced memory scores compared to stimuli uncoupled with the motor system (motor-neutral). Worse memory for motor-neutral stimuli could not be explained by working memory demands (see Experiment $2 b$ ) or task switching (see between-experiment analysis in

Table 3. Memory effects across experiments

\begin{tabular}{|c|c|c|c|c|}
\hline Experiment & $\begin{array}{l}\text { Go vs. No-go } \\
\text { Paired } t \text {-test }\end{array}$ & $\begin{array}{l}\text { Go vs. Motor-neutral } \\
\text { Paired } t \text {-test }\end{array}$ & $\begin{array}{l}\text { No-go vs. Motor-neutral } \\
\text { Paired } t \text {-test }\end{array}$ & $\begin{array}{l}\text { Forced-choice vs. Motor-neutral } \\
\text { Independent } t \text {-test }\end{array}$ \\
\hline 1 & $\begin{array}{l}t(31)=2.81 \\
p=.008\end{array}$ & & & \\
\hline $2 \mathrm{a}$ & $\begin{array}{l}t(31)=0.55 \\
p>.9^{\mathrm{b}}\end{array}$ & $t(31)=3.99, p<.001^{\mathrm{b}}$ & $\mathrm{t}(31)=3.06, p=.013^{\mathrm{b}}$ & \\
\hline $2 b$ & $t(31)=2.68, \quad p=.035^{\mathrm{b}}$ & $t(31)=3.35, p=.006^{\mathrm{b}}$ & $t(31)=0.72, p>.9^{\mathrm{b}}$ & \\
\hline $2 \mathrm{a}$ and $2 \mathrm{~b}$ & $\begin{array}{l}t(63)=2.30 \\
p=.07^{\mathrm{b}}\end{array}$ & $t(63)=5.14, p<.001^{\mathrm{b}}$ & $\begin{array}{l}t(63)=2.90 \\
p=.017^{\mathrm{b}}\end{array}$ & \\
\hline 3 & $\begin{array}{l}t(31)=0.78 \\
p>.9^{\mathrm{b}}\end{array}$ & & & $\begin{array}{l}t(62)=2.63 \\
p=.01\end{array}$ \\
\hline
\end{tabular}

Note. Paired-sample and independent-sample t-tests (two-tailed) comparing memory scores for go versus no-go, go versus motor-neutral, no-go versus motor-neutral, and forced-choice versus motor-neutral. ${ }^{\mathrm{b}}$ Post hoc tests used Bonferroni correction for multiple comparisons 
Experiment 3). Moreover, our findings suggest that engaging the motor system by preparing or executing an action in response to a stimulus enhances the encoding of that stimulus. Considering that Experiment 2a and Experiment 3 used the same number of stimuli and that forced-choice stimuli had better memory scores than motor-neutral stimuli, this also suggests that engaging the motor system may increase the capacity of recognition memory. Interestingly, a similar study (Makovski et al., 2013) found that the motor memory benefit is comparable to a subvocal response (counting) memory benefit. One important design difference to consider is that Makovski et al. (2013; Experiments 1 and 3) used betweenexperiment comparisons of memory scores based on intentional encoding. That is, participants were aware that they would be required to remember the stimuli. In our Experiments, we found that the motor memory benefit extends beyond the memory benefit that may arise from a subvocal response in within- and between-experiment comparisons based instead on incidental encoding. Furthermore, when comparing subvocal responses to motor conditions that included subvocal responses in Experiment 2bExperiment 2b, we still found that memory scores increased as motor engagement increased. In the case of intentional encoding, motorinduced memory benefits, relative to subvocal response memory benefits, may be less pronounced due to a greater emphasis placed on other cognitive processes. Future research is needed to better understand the factors that give rise to the motor-induced encoding effect and memory benefits related to subvocal responses.

Neuroimaging research supports the idea that a neural system involved in motor engagement may signal an influx of activation to support memory encoding. One possible avenue for motor engagement to affect memory encoding is via an attentional network. The pre-SMA, involved in the preparation and execution phase of movements (Luppino, Matelli, Camarda, \& Rizzolatti, 1993), has been shown to correlate with the activity of the dorsal attention network (DAN; Fox et al., 2005). The DAN, involved in regulating the top-down deployment of attention (Vossel, Geng, \& Fink, 2014), has connections with hippocampal regions of the medial temporal lobe (Clower, West, Lynch, \& Strick, 2001; Lavenex, Suzuki, \& Amaral, 2002) and has been shown to modulate memory formation (Cabeza, Ciaramelli, Olson, \& Moscovitch, 2008; Majerus, Péters, Bouffier, Cowan, \& Phillips, 2018; Sestieri, Shulman, \& Corbetta, 2017; Uncapher \& Wagner, 2009). In a go/no-go task context, DAN activation has been associated with stimuli that elicit high motor engagement (Kolodny et al., 2017). In this way, pre-SMA activation that scales as motor engagement increases may reinforce the influence the DAN has on memory encoding. Another possibility is that motor preparation may enhance memory encoding by engaging the noradrenergic system, consistent with the neural network framework provided by Yebra et al. (2019). Although the interaction of neural systems outlined here is speculative, we hypothesize that an attentional network underlies the motor-induced encoding effect. By contrasting the go and no-go conditions with a motor-neutral condition, future neuroimaging work could further elucidate the systems implicated in the present study.

The findings from this study support a framework in which the motor system is situated in cognition, rather than merely serving as an output of cognitive processes. In the current literature, the nature of how motor processes and cognition are related is controversial. Traditional theories of action preparation and action execution describe goal-directed behavior unfolding in a succession of steps: (1) a stimulus is identified and encoded, (2) the abstract representation of the stimulus is compared to internal task goals, (3) decision-making processes evaluate this relationship and which, if any, response is appropriate, and (4) the motor system then executes the selected response (Sternberg, 1969). Similarly, an influential theory, Adaptive Control of Thought-Rational, explains cognitive architecture as being comprised of modules engineered for processes such as visual perception, memory encoding, and motor processes, which are guided by a central procedural component of the frontal cortex to independently interact with other modules (Borst, Nijboer, Taatgen, Van Rijn, \& Anderson, 2015). These theoretical models propose that cognition is disembodied from the motor system. In contrast to these stage-like approaches, cognitive architecture has also been interpreted as an embodied, dynamic system (Thelen \& Smith, 1994; Schöner, Spencer, and DFT Research Group, 2015). Here, action processes are described to coincide with cognitive domains in real-time feedforward and feedback loops (Clark, 1997). This view highlights a bidirectional relationship in which information from the motor and cognitive systems are ever available to each other. That is, the motor system is proposed to be fundamental to cognition.

\section{Conclusion}

In summary, the current study provides evidence that engaging motor processes by preparing or executing actions in response to stimuli facilitate memory encoding and subsequent recognition of those stimuli. Our findings are consistent with an embodied, dynamic systems approach to cognition. This approach has been used to examine the fundamental dependence of cognitive systems on motor activity in the context of reaching behavior (Clearfield, Smith, Diedrich, \& Thelen, 2006; Diedrich et al., 2000; Diedrich, Highlands, Spahr, Thelen, \& Smith, 2001) and spatial memory (Schutte, Spencer, \& Schöner, 2003; Spencer \& Schöner, 2003). These examples highlight the role of acting at spatial locations as a means of influencing memory for spatial locations and subsequent reaching behavior. However, the research presented here expands the scope of these interactions by revealing 
the general influence of motor activity that does not contain stimulus information (e.g., motor movements in space when space is to be remembered). Indeed, our data show that merely preparing or executing an action in the context of perceptual processing of a stimulus enhances the memory of that stimulus. In this way, the present study offers an account of memory in which ongoing motor processes can serve as a valuable component in memory formation. These results expand on avenues of research from an embodied cognition and dynamic systems perspective that can be used to target understanding the bidirectional relationship between the motor system and other cognitive processes.

Acknowledgements We would like to thank A. Caglar Tas for helpful discussion and comments on a prior draft of the manuscript.

Open practices statement The data and materials for all experiments are available upon request to the corresponding author. None of the experiments were preregistered.

\section{References}

Baldauf, D., \& Deubel, H. (2008). Properties of attentional selection during the preparation of sequential saccades. Experimental Brain Research, 184(3), 411-425.

Baldauf, D., Wolf, M., \& Deubel, H. (2006). Deployment of visual attention before sequences of goal-directed hand movements. Vision Research, 46(26), 4355-4374.

Borst, J.P., Nijboer, M., Taatgen, N.A., Van Rijn, H., Anderson, J.R., (2015). Using data-driven model-brain mappings to constrain formal models of cognition. PLOS ONE, 10.

Bosco, A., Daniele, F., \& Fattori, P. (2017). Reaching and grasping actions and their context shape the perception of object size. Journal of Vision, 17(12), 10-10.

Cabeza, R., Ciaramelli, E., Olson, I. R., \& Moscovitch, M. (2008). The parietal cortex and episodic memory: an attentional account. Nature Reviews Neuroscience, 9, 613-625.

Chiu, Y. C., \& Egner, T. (2015a). Inhibition-induced forgetting: when more control leads to less memory. Psychological Science, 26, 2738.

Chiu, Y. C., \& Egner, T. (2015b). Inhibition-induced forgetting results from resource competition between response inhibition and memory encoding processes. Journal of Neuroscience, 35, 11936-11945.

Clark, A. (1997). The dynamical challenge. Cognitive Science: A Multidisciplinary Journal, 21, 461-481.

Clearfield, M. W., Diedrich, F. J., Smith, L. B., \& Thelen, E. (2006). Young infants reach correctly in A-not-B tasks: On the development of stability and perseveration. Infant Behavior and Development, 29(3), 435-444.

Clower, D. M., West, R. A., Lynch, J. C., \& Strick, P. L. (2001). The inferior parietal lobule is the target of output from the superior colliculus, hippocampus, and cerebellum. Journal of Neuroscience, 21(16), 6283-6291.

Davranche, K., Tandonnet, C., Burle, B., Meynier, C., Vidal, F., and Hasbroucq, T. (2007). The dual nature of time preparation: neural activation and suppression revealed by transcranial magnetic stimulation of the motor cortex. The European Journal of Neuroscience $25,3766-3774$.

Diedrich, F. J., Highlands, T. M., Spahr, K. A., Thelen, E., \& Smith, L. B. (2001). The role of target distinctiveness in infant perseverative reaching. Journal of Experimental Child Psychology, 78(3), 263290.

Diedrich, F. J., Thelen, E., Smith, L. B., \& Corbetta, D. (2000). Motor memory is a factor in infant perseverative errors. Developmental Science, 3(4), 479-494.

Ficarella, S. C., \& Battelli, L. (2019). Motor preparation for action inhibition: a review of single pulse tms studies using the go/nogo paradigm. Frontiers in Psychology, 10, 340.

Fox, M. D., Snyder, A. Z., Vincent, J. L., Corbetta, M., Van Essen, D. C., $\&$ Raichle, M. E. (2005). The human brain is intrinsically organized into dynamic, anticorrelated functional networks. Proceedings of the National Academy of Sciences, 102(27), 9673-9678.

Freeman, S. M., and Aron, A. R. (2016). Withholding a reward-driven action: studies of the rise and fall of motor activation and the effect of cognitive depletion. Journal of Cognitive Neuroscience 28, 237 251.

Freeman, S. M., Razhas, I., and Aron, A. R. (2014). Top-down response suppression mitigates action tendencies triggered by a motivating stimulus. Current Biology 24, 212-216.

Fujiyama, H., Hinder, M. R., Schmidt, M. W., Tandonnet, C., Garry, M. I., and Summers, J. J. (2012). Age-related differences in corticomotor excitability and inhibitory processes during a visuomotor RT task. Journal of Cognitive Neuroscience 24, 12531263.

Gutteling, T. P., Kenemans, J. L., \& Neggers, S. F. (2011). Grasping preparation enhances orientation change detection. PLoS One, 6(3).

Hoffman, J. E., \& Subramaniam, B. (1995). The role of visual attention in saccadic eye movements. Perception \& Psychophysics, 57(6), 787795.

Kanade, T., Cohn, J. F., \& Tian, Y. (2000). Comprehensive database for facial expression analysis. Proceedings of the Fourth IEEE International Conference on Automatic Face and Gesture Recognition (FG'00), Grenoble, France, 46-53.

Kiefer, M., Marzinzik, F., Weisbrod, M., Scherg, M., \& Spitzer, M. (1998). The time course of brain activations during response inhibition: evidence from event-related potentials in a go/no go task. Neuroreport, 9(4), 765-770.

Kolodny, T., Mevorach, C., \& Shalev, L. (2017). Isolating response inhibition in the brain: parietal versus frontal contribution. Cortex, 88 , 173-185.

Lavenex, P., Suzuki, W. A., \& Amaral, D. G. (2002). Perirhinal and parahippocampal cortices of the macaque monkey: projections to the neocortex. Journal of Comparative Neurology, 447(4), 394-420.

Lebon, F., Greenhouse, I., Labruna, L., Vanderschelden, B., Papaxanthis, C., and Ivry, R. B. (2015). Influence of delay period duration on inhibitory processes for response preparation. Cerebral Cortex 26, 2461-2470.

Logan, G. D., and Cowan, W. B. (1984). On the ability to inhibit thought and action: a theory of an act of control. Psychological Review 91, 295-327.

Luppino, G., Matelli, M., Camarda, R., \& Rizzolatti, G. (1993). Corticocortical connections of area F3 (SMA-proper) and area F6 (pre-SMA) in the macaque monkey. Journal of Comparative Neurology, 338(1), 114-140.

Ma, Correll, \& Wittenbrink (2015). The Chicago Face Database: A Free Stimulus Set of Faces and Norming Data. Behavior Research Methods, 47, 1122-1135.

Majerus, S., Péters, F., Bouffier, M., Cowan, N., \& Phillips, C. (2018). The Dorsal Attention Network Reflects Both Encoding Load and Top-down Control during Working Memory. Journal of Cognitive Neuroscience, 30(2), 144-159.

Makovski, T., Jiang, Y. V., \& Swallow, K. M. (2013). How do observer's responses affect visual long-term memory?. Journal of Experimental Psychology: Learning, Memory, and Cognition, 39(4), 1097. 
Minear, M., \& Park, D. C. (2004). A lifespan database of adult facial stimuli. Behavior Research Methods, Instruments, \& Computers, 36(4), 630-633.

Miyake, A., Friedman, N. P., Emerson, M. J., Witzki, A. H., Howerter, A., \& Wager, T. D. (2000). The unity and diversity of executive functions and their contributions to complex "frontal lobe" tasks: A latent variable analysis. Cognitive Psychology, 41(1), 49-100.

Naegeli, C., Zeffiro, T., Piccirelli, M., Jaillard, A., Weilenmann, A., Hassanpour, K., ... \& Mueller-Pfeiffer, C. (2018). Locus coeruleus activity mediates hyperresponsiveness in posttraumatic stress disorder. Biological Psychiatry, 83(3), 254-262.

Pavlenko, V. B., \& Kulichenko, A. M. (2003). Self-initiated motor behavioral act-related neuronal activity in the cat locus coeruleus. Neurophysiology, 35(1), 29-37.

Schneider, W. X., \& Deubel, H. (1995). Visual attention and saccadic eye movements: Evidence for obligatory and selective spatial coupling. Studies in Visual Information Processing Vol. 6, 317-324.

Schöner, G., Spencer, J., \& DFT Research Group. (2015). Dynamic Thinking: A Primer on Dynamic Field Theory. Oxford University Press.

Schutte, A. R., Spencer, J. P., \& Schöner, G. (2003). Testing the dynamic field theory: Working memory for locations becomes more spatially precise over development. Child Development, 74(5), 1393-1417.

Sestieri, C., Shulman, G. L., \& Corbetta, M. (2017). The contribution of the human posterior parietal cortex to episodic memory. Nature Reviews Neuroscience, 18, 183-192.

Shepherd, M., Findlay, J. M., \& Hockey, R. J. (1986). The relationship between eye movements and spatial attention. The Quarterly Journal of Experimental Psychology, 38(3), 475-491.

Spencer, J. P., \& Schöner, G. (2003). Bridging the representational gap in the dynamic systems approach to development. Developmental Science, 6(4), 392-412.

Sternberg, S. (1969). The discovery of processing stages: Extensions of Donders' method. Acta Psychologica, 30, 276-315.
Strange, B. A., \& Dolan, R. J. (2004). $\beta$-Adrenergic modulation of emotional memory-evoked human amygdala and hippocampal responses. Proceedings of the National Academy of Sciences, 101(31), 11454-11458.

Tandonnet, C., Garry, M. I., and Summers, J. J. (2010). Cortical activation during temporal preparation assessed by transcranial magnetic stimulation. Biological Psychology 85, 481-486.

Thelen, E, Smith, LB. (1994). A dynamic systems approach to the development of cognition and action. Cambridge, MA: MIT Press

Thomas, S. J., Gonsalvez, C. J., \& Johnstone, S. J. (2009). Sequence effects in the Go/NoGo task: Inhibition and facilitation. International Journal of Psychophysiology, 74(3), 209-219.

Touge, T., Taylor, J. L., and Rothwell, J. C. (1998). Reduced excitability of the corticospinal system during the warning period of a reaction time task. Electroencephalography and Clinical Neurophysiology 109, 489-495.

Uncapher, M. R., \& Wagner, A. D. (2009). Posterior parietal cortex and episodic encoding: insights from fMRI subsequent memory effects and dual-attention theory. Neurobiology of Learning and Memory, 91, 139-154.

Vitrac, C., \& Benoit-Marand, M. (2017). Monoaminergic modulation of motor cortex function. Frontiers in Neural Circuits, 11, 72.

Vossel, S., Geng, J. J., \& Fink, G. R. (2014). Dorsal and ventral attention systems: distinct neural circuits but collaborative roles. The Neuroscientist, 20(2), 150-159.

Yebra, M., Galarza-Vallejo, A., Soto-Leon, V., Gonzalez-Rosa, J. J., de Berker, A. O., Bestmann, S., Oliviero, A., Kroes, M. C. W., \& Strange, B. A. (2019). Action boosts episodic memory encoding in humans via engagement of a noradrenergic system. Nature Communications, 10(1), 1-12.

Publisher's note Springer Nature remains neutral with regard to jurisdictional claims in published maps and institutional affiliations. 